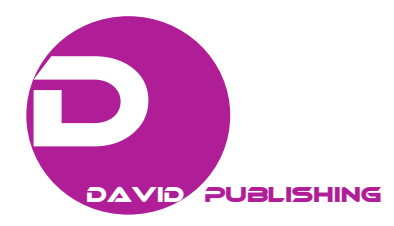

\title{
Using Mixed Reality as a Simulation Tool in Urban Planning Project for Sustainable Development
}

\author{
Hisham El-Shimy ${ }^{1}$, Ghada Ahmed Ragheb ${ }^{1}$ and Amany Ahmed Ragheb ${ }^{2}$ \\ 1. Department of Architectural Engineering, Pharos University, Alexandria 21311, Egypt \\ 2. Department of Architectural Engineering, High Institute of Engineering and Technology, Buhaira 22751, Egypt
}

\begin{abstract}
Nowadays, urban design faces complex demands. It has become a necessity to negotiate between stakeholder objectives, the expectations of citizens and the demands of planning. It is desirable to involve the stakeholders and citizens from an early stage in the planning process to enable their different viewpoints to be successfully expressed and comprehended. Therefore, the basic aim of the study was how the MR (mixed reality) application is designed to encourage and improve communication on urban design among stakeholders and citizens? In this paper, we discuss new approaches to visualize urban building and environment alternatives to different stakeholders and provide them with tools to explore different approaches to urban planning in order to support citizen's participation in urban planning with augmented and mixed reality. The major finding of the study is that learning "how these participatory technologies may help build a community of practice around an urban project". And throughout the different experiences, we can learn to assist towards development of a methodology to use the mixed reality as a simulation tool in the enhancement of collaborative interaction in real-Egyptian project. So, we can determine a number of recommendations to deal with new participatory design tools for urban planning projects.
\end{abstract}

Key words: Mixed reality, real environment, virtual reality, augmented reality, augmented virtuality, MR-tent.

\section{Introduction}

Urban planning is a melting pot for architectural visions in progress. Stakeholders-architects, politicians, citizens and others-bring individual viewpoints into the process. The objective is to refine these viewpoints and achieve mutual consent of all parties [1]. Using virtual/mixed reality environments can minimize problems related to feasibility, experimental control, ethics and cost, but care must be taken to ensure that the environments are immersive and to create "suspension of disbelief" [2].

A commonly used and very inclusive definition of MR (mixed reality) is that, of all applications between pure virtual reality and the real world [3], this definition includes applications where the real environment is mixed with a virtual world. A narrower domain might be considered to be visual output MR

Corresponding author: Hisham El-Shimy, Ph.D., associate professor, research field: sustainable design. E-mail: hisham_elshimy@pua.edu.eg. applications which are focused on mixing the real and the virtual worlds specifically in the visual output device (computer screen, near the eye displays, etc.) as opposed to the mixing of audio or tactile real and virtual worlds.

Using the broad definition of MR, it becomes clear that there is an enormous amount of modes possible for user interaction. Many of the new MR applications are characterized by the blurring of input and output devices (Fig. 1). In MR, the user interaction can be achieved through haptic devices where the position of a finger is tracked in three dimensions and then a haptic feedback device applies the appropriate reverse force (Fig. 2). With mixed reality, we refer to the merging of real and virtual to produce new environments and visualizations.

We have created virtual environments by mixing panoramic imaging and architectural drawings and sketches of future urban plans. With these mixed reality services, we aim to make the plans more visual 


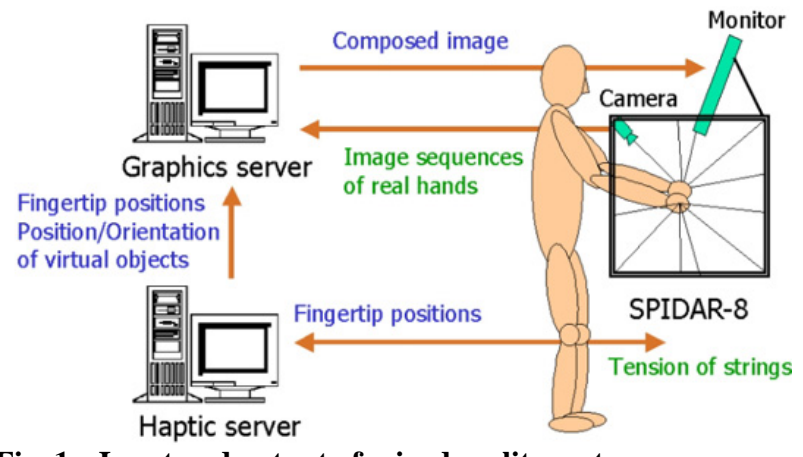

Fig. 1 Input and output of mixed reality system

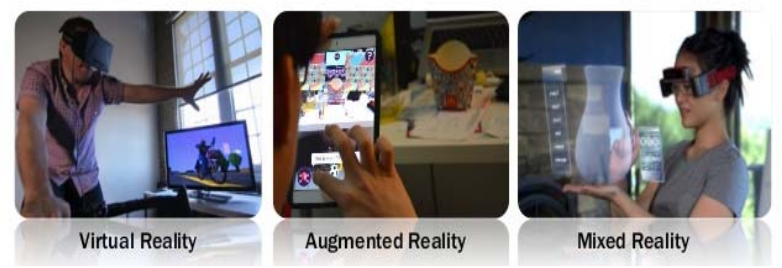

Fig. 2 Tools for making MR.

and understandable to different stakeholders, to be able to discuss the impacts of future urban projects and traffic solutions on their environment at the early stages [4].

By producing easy-to-understand visualizations, it will be possible to view and compare alternative plans and involve citizens and other stakeholders in the planning of the ecology, functionality and quality of their living environments.

\section{Definition of Mixed Reality}

In 1994, Milgram and Kishino [3] defined a mixed reality as "anywhere between the extreme virtuality continuum", where the virtual environment extends from the completely real through to the completely virtual environment with augmented reality and augmented virtuality ranging between. So the mixed reality is a collation of virtual environment, augmented reality and augmented virtuality (Fig. 3).

Urban planning scenes include existing elements which do not change (such as building facades, etc.), but also the elements of the new planned space. In this context, realism includes high-quality geometry and textures for buildings, high-quality models and display for vegetation, accurate and consistent lighting and relighting with shadows, vehicles and traffic simulation, population with individual and crowd animation with simulated behavior, accurate 3D sound rendering, etc. [5].

\section{Using Mixed Reality as a New Approach in Urban Planning}

3D virtual reality is becoming widely used for urban regeneration and planning and transport projects. In urban planning, reaching out and engaging citizens and other stakeholders in making plans are a cornerstone of good practice. The final outcome and plans emerge from the interaction between all the involved stakeholders. There are different ways of demonstrating future urban plans that were introduced. These ways are helpful in figuring out the idea of new visual approaches to community planning and aimed to facilitate feedback related to different approaches.

\subsection{On-site Mixed Reality Mobile Tools}

There are possibilities of visualizing urban planning solutions with smartphones and tablet devices. The idea is for users to be able to move around the surroundings under development and see merged virtual 3D objects and a camera view on a handheld device (Fig. 4). The virtual building objects will be located in their intended locations [6].

\subsection{Interactive Public Screens}

The other presented approach was interactive public screens with mixed reality features (Fig. 5). The screen shows areas under development and new digital visualizations are embedded into the views. Users can manipulate the views and community plan options using their gestures or the touching screen input method. Gesture recognition would be implemented with the help of depth camera sensors. This kind of public screens can be located next to the area [6].

\subsection{Off-site Interactive Design Tables}

The users can explore urban planning solutions using interactive and multiuser design tables (Fig. 6). The tables can be a combination of tangible objects 


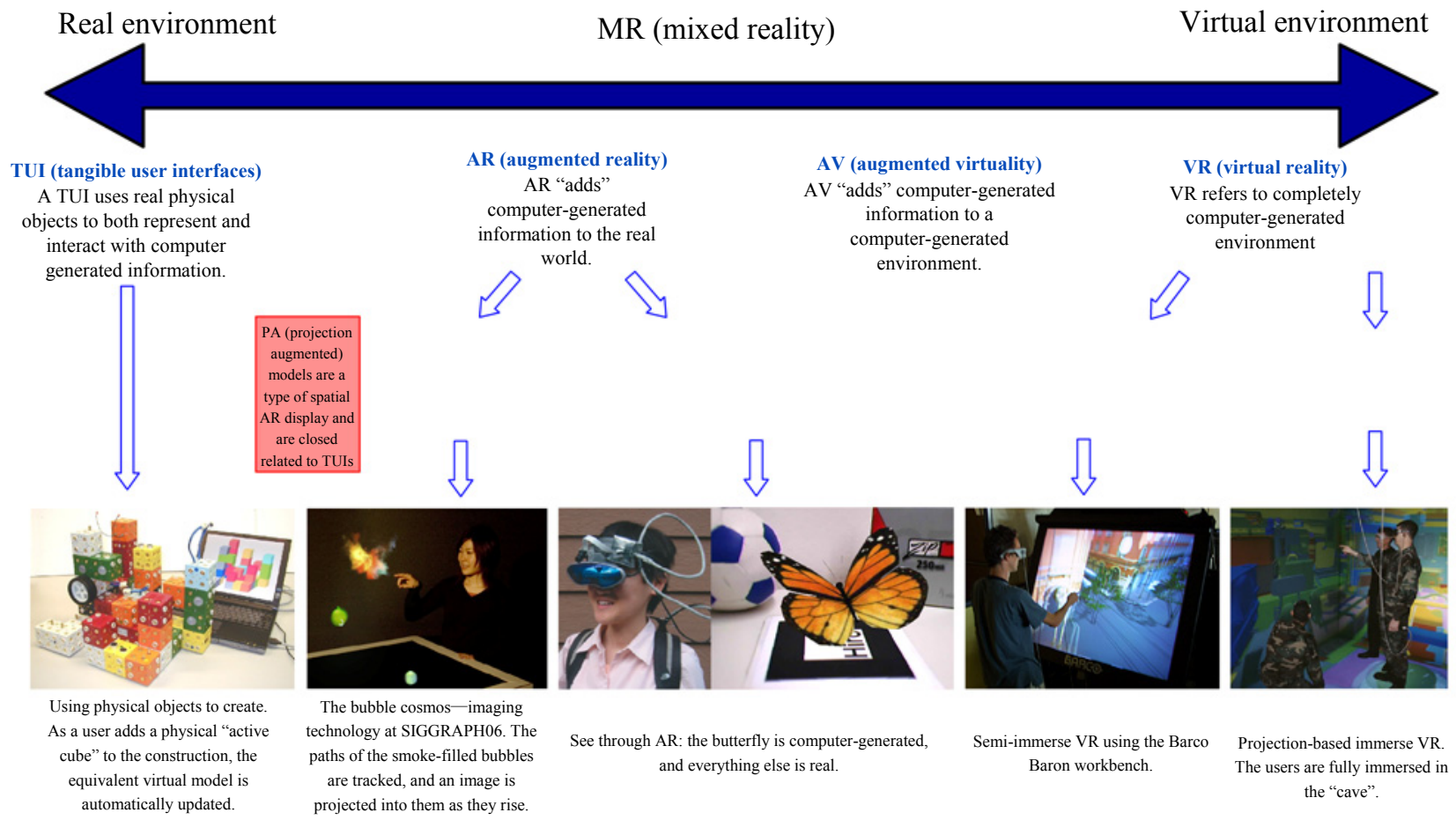

Fig. 3 Output of the type of mixed reality.
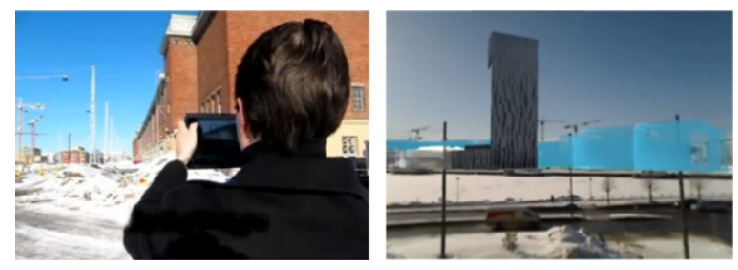

Fig. 4 On-site augmented reality solution.

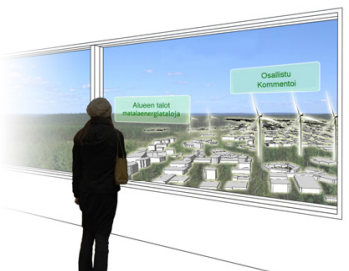

Fig. 5 Concept of an interactive public screen with AR (augmented) features.

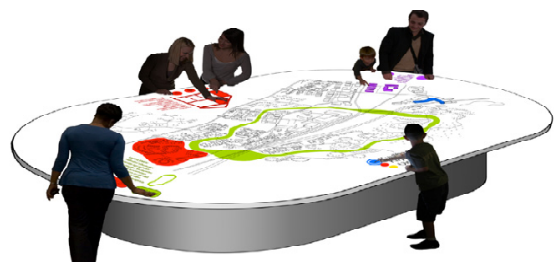

Fig. 6 Visualization on an interactive design table.

or 3D printed building models, projected information and camera recognition systems. The users are able to browse different urban planning options or manipulate objects on a table, and they can receive more information using, e.g., pointing, touching or gestures. The table enables 3D visualizations showing how different buildings look in their environments. The user moves and indicates building options using AR markers on the table [6].

\subsection{MR-Tent}

The idea of the MR-tent is to move out of the laboratory into the field and enable experiments with MR technology right on the site of urban reconstruction. This step is necessary for realizing true AR, so the environment of the real world can be augmented live, in real time, allowing interaction and virtual modification of the MR scene [1]. The technical infrastructure is set up outdoors in the MR-tent (Fig. 7)

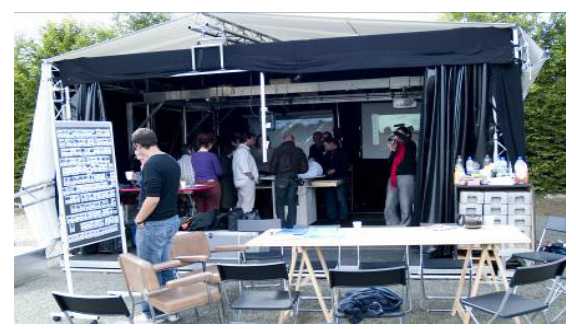

Fig. 7 The MR-tent is a portable lab for using mixed reality in urban planning on location. 
on the site of the urban project. Around the table in the center of the MR-tent, it provides a top view of the MR scene. Users can move and turn objects of different colors and shapes, while an overhead video projection on the table provides interactive feedback. While our previous systems were limited to the detection of positions, colors and sizes, the latest version is able to additionally recognize the shapes and orientations of the colored objects [7].

\subsection{Urban Sketcher}

It is a mixed reality application, which is designed to encourage and improve communication on urban design among stakeholders (Fig. 8). A mix of multimodal input devices enhances collaborative interaction in real-time while visual feedback is given to all participants on a projected live video augmentation from Urban Sketcher, sketching and modifying the scene on site. There is an exchange of information with interactive visual support. This is an instrumental tool for developing visions of future urban spaces by augmenting the real environment with sketches, facades, buildings, green spaces or skylines, a panorama image prepared previously or a direct view seen through a half transparent screen. This see-through screen is made of a white grid providing both a reflective surface for virtual objects and an amount of transparency enabling a view onto the real scene. The multiple interactive views convey and encourage the urban design process [1].

\section{Case Study}

Sidi Gaber railway station is one of the oldest in Egypt. It is the main rail entry point to Alexandria for most travelers. With the new design of Sidi Gaber station project, the need for spatial development becomes a must (Fig. 9). The changes of the train station and the new designed building have caused change in population densities to increase and require spatial development as soon as possible. The organization of this area will allow people to find their way clearly. So, it is important that urban design achieves continuity of the urban fabric and streets to facilitate flows [8]. The purpose of our methodology is to involve citizens in the process of urban planning by providing public installations, placed around a development site, with which anyone may see what the final development will look like and be able to provide direct feedback. It is visualized using mixed reality.

The interviews will find out how stakeholders-architects, politicians, citizens and others, perceive the need to develop current urban planning methods. We can use theme interview method to discuss and collect feedback from new digital visualization and participatory design tool concepts. We want to determine how to support citizens and other stakeholders in involving them in the planning of the sustainability and quality of their living environments through digital services. We want to find out how our service suited this purpose and how to develop it further, especially trying to understand user values, needs and preferences in participative urban planning.

In the interviews, we will present many future urban planning service concepts. Our aim in the interviews will be to place the urban planning concepts in order of importance so that we can choose the kinds of digital urban planning concepts that should be developed in

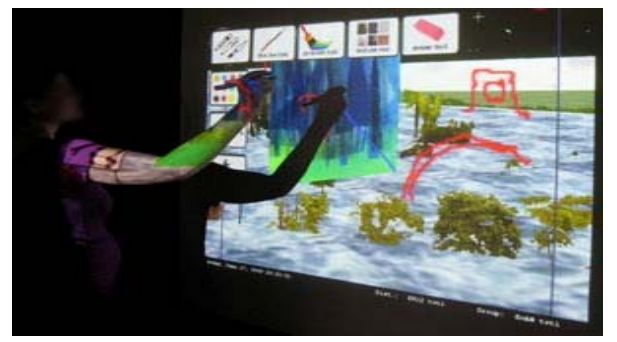

Fig. 8 Sketching on site.

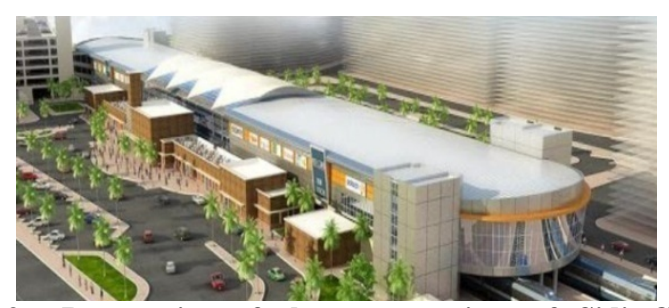

Fig. 9 Perspective of the new project of Sidi Gaber Station. 


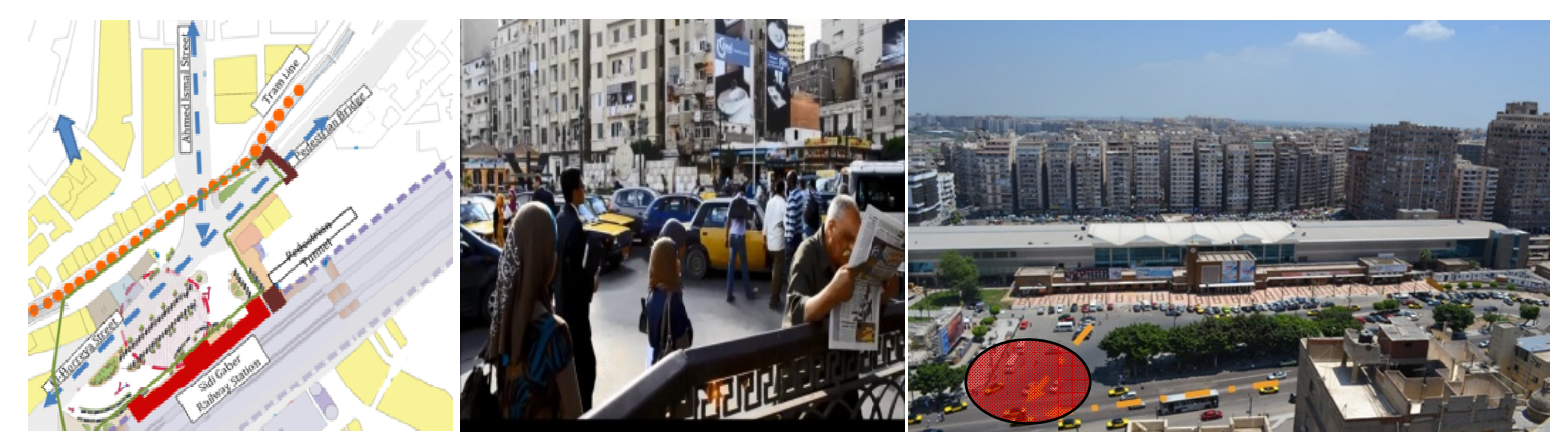

Fig. 10 The current plaza with chaos problem.

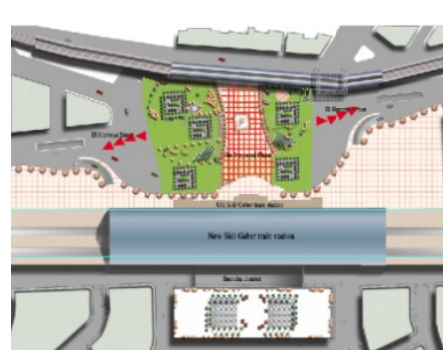

Fig. 11 New model of plaza.

the near future. We also aim, through visualizations, to produce better material for decision-making so that it will be possible to view and compare different options.

Our example will include option for a new design of a plaza in front of the station in order to solve the chaos problem in this plaza. The users were able to investigate the following alternatives: current plaza (Fig. 10).

The new model of plaza (outside the train station until the tram side) allows people to walk or sit with the maximum safety because of landing cars level in the way of Horreya Street under the ground, and raises the level of the tram as a monorail. So, this leads to organize cars circulation and separate between them (Fig. 11). The plaza models will integrate into the panoramic images of the surroundings close to the station building. So, the user can change view freely in panoramic images. Questionnaires will be available on the screen. The existing plaza is architecturally protected and we aim to generate a discussion on the benefits of the future situation.

However, there are many open questions regarding the development of visual, web-based participatory design tools. What kind of things do citizens want to comment on and influence in their living environments? What kind of public projects arouse interest? Are citizens interested in commenting on the new tools which can be used for sustainable urban planning? Will comments and feedback be collected from all possible user segments or mainly or only from the users who are involved in the project in their everyday lives?

We will proceed to end-user trials in real use situations and compare the way citizens and other stakeholders perceive the demonstration system. The piloting phase will also provide an opportunity to analyze differences between on-site and off-site utilization approaches. Technically, the system could be developed to be more location- and augmented-reality-oriented, which means that the architectural 3D models could be automatically adapted to the real-time camera views. The objects on the screen could be also interactive, and users could add comments or fill out questionnaires by selecting preferred design solutions in the view. Digitizing information and increasing on-line channels are the most powerful way to facilitate access to information and make the recent closed urban planning processes more open and participative. So, the augmented reality 
solution allows the stakeholders to view and compare digital models of buildings on their proposed location. Moreover, like a city or a house, the MR-tent is a place that stimulates the desire to be together and invites a common decision: a place where people can meet, discover and appropriate a world, in other words, to live together inside a complex and sometime contradictory community. The prototype of Urban Sketcher is an MR application supporting a range of devices for collaborative multi model interaction.

\section{Conclusions}

In this paper, we can learn from different experiences to assist towards development of a methodology to engage real users in the process of design and evaluation for real-world. Mixed reality is an inelegant tool for urban planning. Its technologies can be used for participatory urban planning and co-creation of future living environments with different stakeholders. More open discussions with different stakeholders, illustrating and visualizing urban plans, will enhance the quality of the decision-making materials. The new tools of mixed reality will make it possible to illustrate and compare different options and their direct and indirect impacts on the environment. In addition, they will offer users the option to give feedback and share their ideas at any time of the day they want. A good option to demonstrate future urban plans to different stakeholders is lightweight mobile solutions, which can be taken to different places and situations at any time and used to illustrate alternatives. More demanding approaches, such as interactive design tables, can be also useful, especially for large urban planning projects.

The public display boards were seen as effective marketing methods for new urban plans. However, as long as the system is located in a public place and close to people flows, it will have an effect on participation by shy or privacy-oriented people. The actual participation, e.g., responding to surveys, sharing ideas and feedback, would happen most conveniently with a personal mobile or other personal devices. Citizens in general are interested in commenting on and participating in urban planning projects, which are related to their everyday lives and their own neighborhood. Afterwards, citizens should be informed, for instance, that answering the survey was useful and that their feedback has been taking into consideration in the urban planning. There are currently no proper tools for this.

\section{References}

[1] Sareika, M., and Schmalstieg, D. 2007. Urban Sketcher: Mixed Reality on Site for Urban Planning and Architecture. Graz: Graz University of technology.

[2] Park, A. J., Calvert, T. W., and Brantingham, P. J. 2008. "The Use of Virtual and Mixed Reality Environments for Urban Behavioral Studies." Psychology Journal 6 (2): 119-30.

[3] Milgram, P., and Kishino, A. F. 1994. "Taxonomy of Mixed Reality Visual Displays." IEICE (Institute of Electronic, Information, and Communication Engineers) Transactions on Information and Systems 77 (12): 1321-9.

[4] Merrill, K., and Maes, P. 2007. "Siftables: Towards Sensor Network User Interfaces." In Proceedings of the 1st International Conference on Tangible and Embedded Interaction, 75-8.

[5] Drettakis, G., Roussou, M., Reche, A., and Tsingos, N. 2006. "Design and Evaluation of a Real-World Virtual Environment for Architecture and Urban Planning." Teleoperators and Virtual Environments 16 (3): 318-32.

[6] Oksman, V., Väätänen, A., Ylikauppila, M. 2014. "Future Illustrative and Participative Urban Planning Developing Concepts for Co-creation." Presented at the 6th International Conference on Creative Content Technologies VTT Technology Centre of Finland, Tampere, Finland.

[7] Maquil, V., Sareika., M., Schmalstieg, D., and Wagner, I. 2009. "MR-Tent: A Place for Co-constructing Mixed Realities in Urban Planning." In Proceedings of Graphics Interface 2009, 211-4.

[8] Sharaf, S. H., and Ragheb, G. H. 2013. "Strengthening Alexandria Urban Fabric by Planning Urbanism's Walkable Area." In Proceedings of the 18th international Conference on Urban Planning and Regional Development in the Information Society in Italy. (CD-ROM) 